\title{
Ascodipteron sanmingensis sp. nov., a new bat fly (Hippoboscidae: streblid grade) from Fujian, China
}

\author{
Haoran Sun $\ddagger$, Liang Ding§, Liping Yan${ }^{\ddagger}$, Thomas Papel, Dong Zhang ${ }^{\ddagger}$ \\ $\ddagger$ School of Ecology and Nature Conservation, Beijing Forestry University, Beijing, China \\ $\S$ Go with KIDS Natural History Workshop, Beijing, China \\ | Natural History Museum of Denmark, Copenhagen, Denmark
}

Corresponding author: Dong Zhang (ernest8445@163.com)

Academic editor: Pierfilippo Cerretti

Received: 17 Feb 2021 | Accepted: 10 Apr 2021 | Published: 23 Apr 2021

Citation: Sun H, Ding L, Yan L, Pape T, Zhang D (2021) Ascodipteron sanmingensis sp. nov., a new bat fly

(Hippoboscidae: streblid grade) from Fujian, China. Biodiversity Data Journal 9: e64558.

https://doi.org/10.3897/BDJ.9.e64558

ZooBank: urn:Isid:zoobank.org:pub:E09AF2EA-BE79-454A-82F9-C064DE8CD396

\begin{abstract}
\section{Background}

The bat fly genus Ascodipteron Adensamer, 1896 currently contains 15 species, all of which occur in tropical and subtropical areas of the Eastern Hemisphere. A new species of endoparasitic bat fly, Ascodipteron sanmingensis sp. nov., was collected from the Great Himalayan Leaf-nosed Bat, Hipposideros armiger (Hodgson, 1853), during ecological studies on bats in Fujian, China.
\end{abstract}

\section{New information}

A new species, Ascodipteron sanmingensis sp. nov., is described, based on dealate neosomic females and is supported by molecular data from a $368 \mathrm{bp}$ fragment of the cytochrome B (Cytb) gene. Habitus and diagnostic details, as well as the attachment sites on the host, are documented with photographs. A detailed comparison of the new species with related species is provided and the new species is accommodated in the most recent key to the world species of Ascodipteron. 


\section{Keywords}

Ascodipterinae, dealate neosomic female, endoparasite, Great Himalayan Leaf-nosed Bat

\section{Introduction}

Bats are parasitised by some 500 species of hippoboscoid bat flies (Dick and Patterson 2006). These have traditionally been classified in the families Streblidae and Nycteribiidae, but increasing phylogenetic evidence suggests that the Streblidae are paraphyletic, with the monophyletic Nycteribiidae (which is the older name) nested inside an Old World 'streblid grade' (Petersen et al. 2007, Dittmar et al. 2015, Dittmar et al. 2006, Pape et al. 2011). Within this streblid grade, the Ascodipterine bat flies are highly specialised endoparasites, easily distinguishable from other streblids by their unusual mode of parasitic life and strong polymorphism in the adult stage, where females lose halteres, wings and legs at the trochanter immediately after attachment to a host bat and transform into neosomes (Hastriter and Bush 2006, Hastriter 2007). Apart from the monotypic genus Maabella Hastriter \& Bush, 2006, all other ascodipterines are assigned to the genus Ascodipteron Adensamer, 1896 and the distribution of diagnostic features would appear to support that the two taxa most likely are sister taxa (Hastriter and Bush 2006, Hastriter 2007).

Early taxonomists, such as Adensamer (1896), Muir (1912), Monticelli (1898), Speiser (1908), Jobling (1939), Jobling (1940), Jobling (1952), Jobling (1956), Jobling (1958) and Lavoipierre (1946), studied Ascodipteron spp. from the Afrotropical Region, which formed the basis for further research on this genus and Maa (1965) revised the African species for the first time. Hastriter and Bush (2006) erected the genus Maabella and suggested Paraascodipteron Advani and Vazirani, 1981 should be placed in a different subfamily. Following these advances, Hastriter (2007) presented a worldwide species list, a key to all Ascodipterinae and a taxonomic review of the Oriental and Australasian species including what is known on their biology.

During the course of examining parasites collected from bats in Fujian, China, we found dealate females (neosomes) of one further undescribed species of Ascodipteron, which is morphologically similar to $A$. phyllorhinae Adensamer, 1896 and $A$. speiserianum Muir, 1912. Despite the flood of molecular data in the phylogenomic era (Misof et al. 2014, Kutty et al. 2019, Yan et al. 2020), morphological characters are still essential in phylogenetic and evolutionary studies (Giribet 2015). Therefore, the purpose of our study is to describe the new species and report on the host species and site of attachment and compare the new species with its most similar congeners, which will extend our knowledge of the taxonomy, distribution and biology of these parasitic flies. 


\section{Materials and methods}

\section{Sample collection}

During October and November 2020, 10 bat flies were collected from a colony of Hipposideros armiger (Hodgson, 1853) roosting in an abandoned bomb shelter in a residential area of Qunying Second Village, Meilie District, Sanming, Fujian, China ( $26^{\circ} 16^{\prime} 30.33^{\prime \prime} \mathrm{N}, 117^{\circ} 36^{\prime} 49.02^{\prime \prime} \mathrm{E} ; 195$ m a.s.I.).

Entire female ascodipterine bat flies (neosomes) were removed with forceps without hurting the host. Three specimens were preserved in $75 \%$ ethanol and seven in $95 \%$ ethanol, all deposited at the Museum of Beijing Forestry University, Beijing, China (MBFU).

\section{Specimen imaging, measurements and terminology}

Z-stack photographs were acquired with a Zeiss Axio Zoom.V16 microscope (Carl Zeiss AG, Oberkochen, Germany) equipped with a PlanApo Z 1.0×/0.25 FWD 60 objective and an AxioCam 503 colour camera. Images were processed with the software Zen 2 and Adobe Photoshop CS6 (Adobe Systems Incorporated, San Jose, USA) by cropping, contrast enhancement and removal of the background.

Ecological photographs were taken with EF $100 \mathrm{~mm}$ f/2.8L IS USM and MP-E $65 \mathrm{~mm}$ f/2.8 1-5X lenses attached to a Canon 5D Mark IV SLR camera. Images and plates were processed on a standard Windows 10 platform using Adobe Photoshop CS6 (Adobe Systems, Inc., San Jose, CA, USA).

Measurements and terminology follow Hastriter and Bush (2006).

\section{DNA extraction, amplification, sequencing and sequence editing}

One specimen (BFU-2227) of Ascodipteron sanmingensis sp. nov. was selected for DNA extraction. The specimen was dissected and abdominal muscle tissue was used to extract the total genomic DNA, using the DNeasy Blood \& Tissue kit (Qiagen, Dusseldorf, Germany). The remaining body parts were retained as vouchers and deposited in the entomological collection of Beijing Forestry University. A fragment of $368 \mathrm{bp}$ of the cytochrome B (Cytb) gene was amplified using the primer pairs A5 (forward: 5'-AGG RCA AAT ATC ATT TTG AG-3') and B1.1 (reverse: 5'-AAA TAT CAT TCT GGT TGA ATA TG-3') (Dittmar and Whiting 2003). PCR reactions were conducted as described in Zhang et al. (2016) and Yan et al. (2019) and amplification conditions as described by Dittmar and Whiting (2003). The PCR products were purified and sequenced bidirectionally by BGI Inc., Beijing, China.

SeqMan Pro v. 7.1.0 (DNASTAR Inc., USA) was used to edit and assemble the forward and reverse sequences. 


\section{DNA sequence analysis}

We downloaded the only two mitochondrial cytochrome b gene (Cytb) sequences of the genus Ascodipteron from GenBank. The sequences, together with the Cytb sequenced in this study, were aligned using Muscle as implemented in Mega X (Kimura 1980, Kumar et al. 2018). Subsequently, nucleotide sequence divergences were calculated, using Kimura 2-parameter (K2P) model in Mega X.

\section{Taxon treatment}

\section{Ascodipteron sanmingensis, sp. $\mathrm{n}$.}

- ZooBank 7CB73EAD-4A34-4357-93A8-302A0B5E4462

\section{Materials}

\section{Holotype:}

a. scientificName: Ascodipteron sanmingensis; phylum: Arthropoda; class: Insecta; order: Diptera; family: Streblidae; genus: Ascodipteron; specificEpithet: sanmingensis; continent: South China; country: China; countryCode: CN; stateProvince: Fujian; county: Sanming; locality: Meilie District; verbatimLocality: Qunying Second Village; verbatimElevation: 195

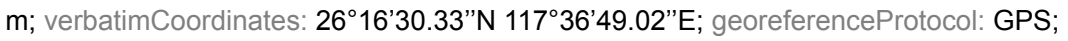
samplingProtocol: Entire neosomes were removed with forceps from the bats; eventDate: Oct-2020; individualID: BFU-2230; individualCount: 1; sex: female; lifeStage: adult; recordedBy: Liang Ding; language: en; institutionID: the Museum of Beijing Forestry University, Beijing, China; institutionCode: MBFU; collectionCode: Insects; basisOfRecord: PreservedSpecimen

\section{Paratypes:}

a. scientificName: Ascodipteron sanmingensis; phylum: Arthropoda; class: Insecta; order: Diptera; family: Streblidae; genus: Ascodipteron; specificEpithet: sanmingensis; continent: South China; country: China; countryCode: $\mathrm{CN}$; stateProvince: Fujian; county: Sanming; locality: Meilie District; verbatimLocality: Qunying Second Village; verbatimElevation: 195 m; verbatimCoordinates: 26¹6'30.33”N 117'36'49.02”E; georeferenceProtocol: GPS; samplingProtocol: Entire neosomes were removed with forceps from the bats; eventDate: Oct-2020; individualID: BFU-2225, BFU-2226, BFU-2227, BFU-2228, BFU-2229,; individualCount: 5; sex: female; lifeStage: adult; recordedBy: Liang Ding; dateldentified: 2020; language: en; institutionID: the Museum of Beijing Forestry University, Beijing, China; institutionCode: MBFU; collectionCode: Insects; basisOfRecord: PreservedSpecimen

b. scientificName: Ascodipteron sanmingensis; phylum: Arthropoda; class: Insecta; order: Diptera; family: Streblidae; genus: Ascodipteron; specificEpithet: sanmingensis; continent: South China; country: China; countryCode: CN; stateProvince: Fujian; county: Sanming; locality: Meilie District; verbatimLocality: Qunying Second Village; verbatimElevation: 195 m; verbatimCoordinates: 26¹6'30.33"N 117³6'49.02"E; georeferenceProtocol: GPS; samplingProtocol: Entire neosomes were removed with forceps from the bats; eventDate: 11/15/2020; individualID: BFU-2221, BFU-2222; individualCount: 2; sex: female; lifeStage: adult; recordedBy: Liang Ding \& Hao-Ran Sun; dateldentified: 2020; language: 
en; institutionID: the Museum of Beijing Forestry University, Beijing, China; institutionCode: MBFU; collectionCode: Insects; basisOfRecord: PreservedSpecimen c. scientificName: Ascodipteron sanmingensis; phylum: Arthropoda; class: Insecta; order: Diptera; family: Streblidae; genus: Ascodipteron; specificEpithet: sanmingensis; continent: South China; country: China; countryCode: CN; stateProvince: Fujian; county: Sanming; locality: Meilie District; verbatimLocality: Qunying Second Village; verbatimElevation: 195 m; verbatimCoordinates: 26¹6'30.33”N 117 $36^{\circ} 49.02$ 'E; georeferenceProtocol: GPS; samplingProtocol: Entire neosomes were removed with forceps from the bats; eventDate: 11/17/2020; individualID: BFU-2223, BFU-2224; individualCount: 2; sex: female; lifeStage: adult; recordedBy: Liang Ding \& Hao-Ran Sun; dateldentified: 2020; language: en; institutionID: the Museum of Beijing Forestry University, Beijing, China; institutionCode: MBFU; collectionCode: Insects; basisOfRecord: PreservedSpecimen

\section{Description}

Female. Head. Labial theca slightly longer than wide (Fig. 2B,D; Fig. 3B,C); posterior margin concave dorsally, convex ventrally. Labial theca dorsally with ca. $50+$ pigmented, peg-like, spiniform setae and ventrally with ca. $100+$ similar setae. Peglike setae identical to those on gena. Gena with ca. 40 peg-like setae on dorsal half; anterior margin convex (Fig. $3 \mathrm{~A}$ ). Posterior margin of frons trilobed. Arista with multiple fine branches; basal antennal segment with a single long seta. Lateral vertex with convex antero-medial margin; adorned with 30-36 long setae; about twice as long as wide, with a longitudinal fold in the lateral portion of the sclerite (Fig. 4A). Occipital sclerite triangular (Fig. 2B).

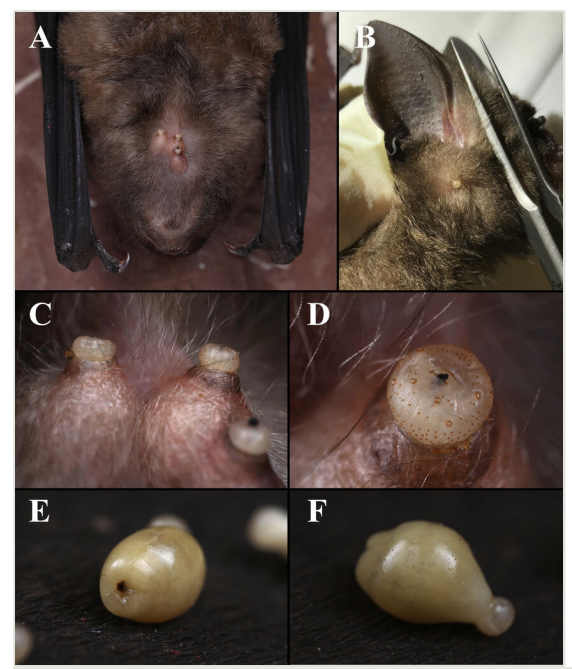

Figure 1. doi

Ascodipteron sanmingensis sp. nov. and its host Hipposideros armiger. A. Neosomes protruding from the lower jaw area of host. B. Neosomes protruding from the base of an ear of host. C-D. Neosomes embedded in host tissue. E-F. Whole neosomes freshly extracted from host (head and thorax fully withdrawn). 


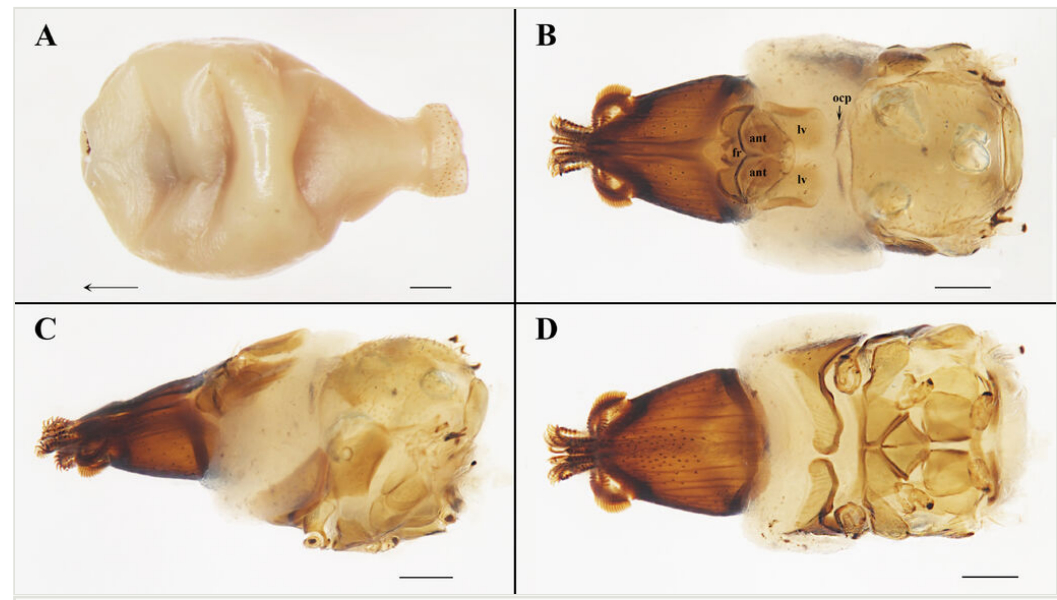

Figure 2. doi

Ascodipteron sanmingensis sp. nov., ex. H. armiger, China. A. Whole neosome (head and thorax fully withdrawn, the arrow indicates the direction of the head) (BFU-2227, paratype). B-D. Head and thorax (BFU-2228, paratype); dorsal view (B), lateral view (C) and ventral view (D). Abbreviations: ant, antenna; fr, frons; Iv, lateral vertex; and ocp, occipital sclerite. Scale bars: $A=500 \mu \mathrm{m} ; B-C=200 \mu \mathrm{m}$.
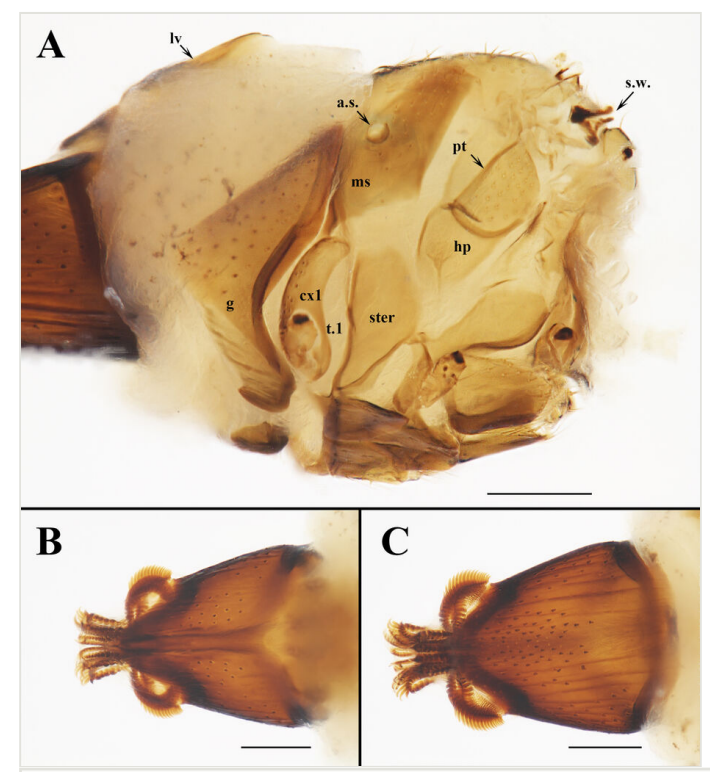

Figure 3. doi

Ascodipteron sanmingensis sp. nov., ex. H. armiger, China (BFU-2228, paratype). A. Thorax, lateral dorsal view. B. Labial theca, dorsal view. C. Labial theca, ventral view. Abbreviations: a.s., anterior thoracic spiracle; cx1, coxa 1; g, gena; hp, hypopleuron; Iv, lateral vertex; ms, mesopleuron; pt, pteropleuron; s.w., stump of wing; ster, sternopleuron; and t.1, trochanter 1. Scale bars: $A-C=200 \mu \mathrm{m}$. 


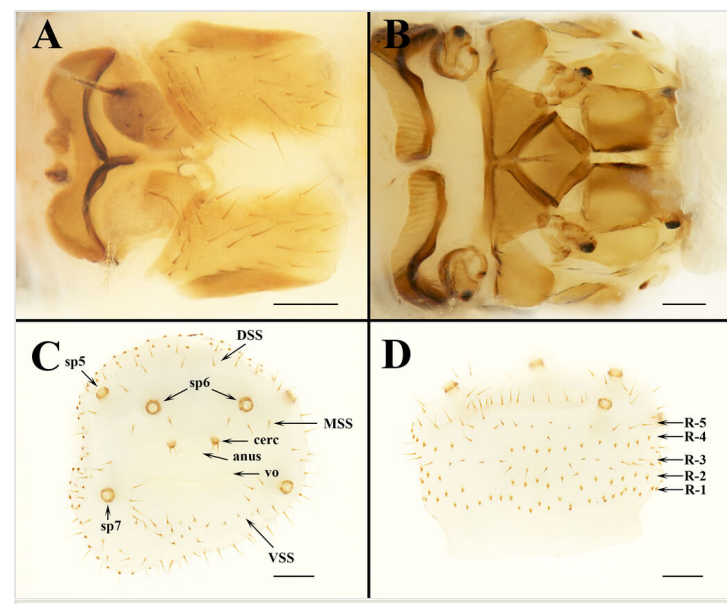

Figure 4. doi

Ascodipteron sanmingensis sp. nov., ex. H. armiger, China. A. Frons and lateral vertex (BFU2221, paratype). B. Thorax, ventral view (BFU-2221, paratype). C. Abdomen, posterior view (BFU-2223, paratype). D. Five terminal annular rows of setae (BFU-2223, paratype). Abbreviations: sp5-sp7, spiracles 5-7; DSS, dorsal spiracular setae; MSS, medial spiracular setae; VSS, ventral spiracular setae; anus, anus; cerc, cercus; vo, vaginal orifice; R1-5, abdominal setae arranged roughly into annular rows comprised of variable types of setae, R1 the proximal and R5 the distal row. Scale bars: $A-B=100 \mu \mathrm{m} ; C-D=200 \mu \mathrm{m}$.

Thorax(Fig. 2B,D; Fig. 3A; Fig. 4B). Scutum with numerous long setae, especially numerous along posterolateral margin; devoid of setae along mid-line. Mesopleuron with 8-10 short, peg-like spiniform setae anterior to large round spiracle; setae posterior to spiracle of three varieties: 6-7 short, peg-like, spiniform setae (ventral); 6 8 longer, sharp, spiniform setae (medial); 8-10 long, slender setae (along posterior margin and dorsal). Pteropleuron with 20-26 short, peg-like, spiniform setae in dorsal half of sclerite; ventral half devoid of setae. Hypopleuron and sternopleuron without setae. Coxa 1 with 12-15 peg-like, spiniform setae and 3 long, slender setae on proximal half. Coxae 2 and 3 each with a cluster of 8-11 long, slender setae. Trochanters 1 and 2 each with 2-3 minute, spiniform setae on anterior apex; 1-2 slender setae on posteroapical margin. Prosternum devoid of setae, mesosternum with 2-3 slender setae, metasternum with 4 short setae and posterolateral margin distinctly concave.

Genital Aperture (Fig. 4C and D). Five terminal annular rings arranged roughly into rows comprised of different types of setae (abbreviated R1-5, with R1 the proximal and R5 the distal row). R1, R2 and R4 with minute, thick setae; R3 and R5 with long setae. VSS (ventral spiracular setae situated in a ventral, arching row or grouping between spiracles \#7) of 19-23 long setae. MSS (medial spiracular setae) arranged in symmetrical groups of 2-3 setae, situated between spiracles \#6 and \#7. DSS (dorsal spiracular setae) arranged in a single dorsal arching row of 7-8 long setae between spiracles \#5. Cercus with 4-6 setae, diameter ca. $43 \mu \mathrm{m}$. 
Dimensions. Head and thorax: $1671 \mu \mathrm{m}(\mathrm{n}=3$, range: 1666-1676 $\mu \mathrm{m})$; Labial theca, length: $626 \mu \mathrm{m}(\mathrm{n}=2$, range: 556-695 $\mu \mathrm{m})$; width: $511 \mu \mathrm{m}(\mathrm{n}=2$, range: $495-526 \mu \mathrm{m})$, genital aperture, diameter: $1171 \mu \mathrm{m}(\mathrm{n}=2$, range: $1125-1217 \mu \mathrm{m})$, neosome, length: $4655 \mu \mathrm{m}$.

Male. Unknown.

\section{Diagnosis}

Labial theca slightly longer than wide (Fig. 2B and D; Fig. 3B and C), R1, R2, R4 as minute, thick setae and R3, R5 as long setae. Most similar to $A$. phyllorhinae and $A$. speiserianum, but separable from the former by having 50 + spiniform setae dorsally on the labial theca and a lateral fold on the lateral vertex and from the latter by the setose lateral vertex (Fig. 4A).

\section{Etymology}

The new species is named after its type locality Sanming.

\section{Distribution}

Oriental - China (Fujian).

\section{Biology}

Ascodipteron sanmingensis sp. nov. is embedded at the base of an ear or on the lower jaw area of Hipposideros armiger (Fig. 1A-D). The neosomes are not inserted perpendicular to the skin, but at a slight angle.

\section{Molecular results}

The genetic distance between Ascodipteron sanmingensis sp. nov. and A. phyllorhinae is $7.75 \%$ and between $A$. sanmingensis sp. nov. and an unidentified $A$. sp. is $11.86 \%$ (Table 1). The Cytb sequence generated in this study was deposited in GenBank (Accession: MW822598).

\section{Table 1.}

Pairwise differences of mitochondrial cytochrome b gene (Cytb) sequences between species, based on Kimura 2-parameter

\begin{tabular}{|l|l|l|l|}
\hline & & 1 & 2 \\
\hline 1 & Ascodipteron sanmingensis sp. nov. (MW822598) & & \\
\hline 2 & Ascodipteron phyllorhinae (DQ133149.1) & 0.0775 & \\
\hline 3 & Ascodipteron sp. (DQ133154.1) & 0.1186 & 0.1057 \\
\hline
\end{tabular}




\section{Identification keys}

\section{Remarks:}

Ascodipteron sanmingensis sp. nov. will run to couplet 14 in the identification key to dealate ascodipterine females proposed by Hastriter (2007) and it can be incorporated in the key as follows, with host data given in square brackets:

1

Medial spiracular setae (MSS) comprised of two or three setae. First abdominal annular row (R1) present.

Medial spiracular setae (MSS) comprised of two setae grouped adjacent to

spiracle \#6. First abdominal annular row (R1) absent. [Rhinolophus, Africa.]

Labial theca dorsally with 25-30 lightly pigmented peg-like spiniform setae.

2 Lateral vertex without fold or reinforcement in the lateral portion of the sclerite. [Hipposideros spp., usually on wing, SE China to Solomon Islands.]

Labial theca dorsally with ca. $50+$ pigmented peg-like spiniform setae. Lateral vertex with a longitudinal fold or reinforcement in the lateral portion of the sclerite. [Hipposideros armiger, at the base of ear or on the lower jaw area, only known from Fujian, China.]
2

A. brevior

A. phyllorhinae

A. sanmingensis sp. nov.

\section{Discussion}

Hastriter (2007) revised the species of Ascodipteron from the Oriental and Australasian regions and provided a detailed review of what is known of their biology. He studied extensive material of $A$. phyllorhinae from across its extensive range and he mentioned that the specimens "may prove to represent more than one species" (p. 13). Given the very small morphological differences between $A$. phyllorhinae and $A$. sanmingensis sp. nov., a closer examination of the lectotype of $A$. emballoneurae Banks from Borneo would seem justified.

Only two other species of Ascodipteron are, so far, known to use Hipposideros armiger as host: $A$. longiascus Hastriter, 2007, known in four specimens obtained from a single specimen of $H$. armiger in China (Yunnan) and $A$. phyllorhinae, which appears to favour $H$. diadema Geoffroy, 1813 as its host (Hastriter 2007).

Ascodipteron sanmingensis sp. nov. is only known from China (Fujian), although its known host has a much wider distribution over most of South-East Asia (Bates et al. 2020). Hastriter (2007) concluded that "Of the hundreds of specimens studied across the entire Oriental and Australasian regions ... there are only two previously described sympatric Ascodipteron species, A. phyllorhinae and A. speiserianum". Both A. phyllorhinae and A. speiserianum have broad distributional ranges, but are, in general, of a more southern distribution, with $A$. phyllorhinae reaching north into southern China (Guangxi) and $A$. 
speiserianum reaching further north into China (Hainan, Taiwan) and southern Japan (Hastriter 2007). Further sampling is needed to fully assess the degree of sympatry for these three species.

Liu et al. 2018 evaluated the potential use of Cytb for insect species identification and found it almost as efficient as $\mathrm{CO}$ with a success rate of $95 \%$ correct identifications. (Gilarriortua et al. 2013, Liu et al. 2018).

Dittmar et al. 2006 provided molecular data from two species of Ascodipteron from a single locality in Malaysia, Pahang. These were identified as A. phyllorhinae and a species given as new to science, but not described. Comparing data from the present material with Cytb sequences provided by Dittmar et al. 2006 reveals a difference of $7.75 \%$ with their $A$. phyllorhinae and of $11.86 \%$ with the species considered to be undescribed (Table 1). The intraspecific variation of Cytb for insects has been estimated to be $0.66 \pm 0.81 \%$ by Liu et al. (2018) in able 2 , indicating that $A$. sanmingensis sp. nov. is a different species from $A$. phyllorhinae and $A$. sp.

\section{Acknowledgements}

We should like to express our sincere thanks to Dr. Michael W. Hastriter (Monte L. Bean Life Science Museum, Brigham Young University, Provo, Utah, USA), who generously provided invaluable information about ascodipterine bat flies.

We wish to thank Mr. Liang Guo and Mr. Taokun Liao, both of Sanming City, for providing access to the abandoned bomb shelter and assisting in collecting specimens. Thanks also to Mr. Mingyuan Fan (PhD student at China Agricultural University) for his assistance in collecting specimens. Special thanks to Mr. Siyao Huang (South China Agricultural University) for kind suggestions for photographing specimens preserved in ethanol. This study was carried out with financial support from the National Natural Science Foundation of China (no. 31872964) and Fundamental Research Funds for the Central Universities (no. 2019JQ0318) to D.Z., China National Postdoctoral Program for Innovative Talents (no. BX20190042) and China Postdoctoral Science Foundation (no. 2020M670177) to L.Y.

\section{Author contributions}

Liang Ding and Hao-ran Sun collected materials of Ascodipteron sanmingensis sp. nov. and provided all the photos. Hao-ran Sun and Liping Yan obtained and analysed molecular data. All authors analysed the morphological data, wrote the description and contributed to the discussion.

Haoran Sun and Liang Ding contributed equally to this study. 


\section{References}

- $\quad$ Adensamer T (1896) Über Ascodipteron phyllorhinae (n. gen. n. sp.), eine eigenthümliche pupiparenform. Sitzungberichte der Akademie der Wissenschaften in Wien. Mathematisch-naturwissenschaftliche Klasse 105: 400-416.

URL: https://www.biodiversitylibrary.org/page/35613378\#page/442/mode/1up

- Bates PJ, Bumrungsri S, Francis C, Csorba G, Oo SS (2020) Hipposideros armiger. The IUCN Red List of Threatened Species 2020: e.T10110A22097743.

https://dx.doi.org/10.2305/IUCN.UK.2020-2.RLTS.T10110A22097743.en

- Dick CW, Patterson BD (2006) Bat flies-obligate ectoparasites of bats. In: Morand S, Krasnov BR, Poulin R (Eds) Micromammals and Macroparasites. Springer-Verlag, Tokyo, xv + 647 pp. [ISBN 978-4-431-36025-4]. https://doi.org/10.1007/9784-431-36025-4

- Dittmar K, Whiting MF (2003) Genetic and phylogeographic structure of populations of Pulex simulans (Siphonaptera) in Peru inferred from two genes (CytB and Coll). Parasitology Research 91: 55-59. https://doi.org/10.1007/s00436-003-0879-5

- Dittmar K, Porter ML, Murray S, Whiting MF (2006) Molecular phylogenetic analysis of nycteribiid and streblid bat flies (Diptera: Brachycera, Calyptratae): implications for host associations and phylogeographic origins. Molecular Phylogenetics and Evolution 38 (1): 155-170. https://doi.org/10.1016/j.ympev.2005.06.008

- Dittmar K, Morse S, Dick C, Patterson B (2015) Bat fly evolution from the Eocene to the present (Hippoboscoidea, Streblidae and Nycteribiidae parasite diversity and diversification: Evolutionary ecology meets phylogenetics. In: Morand S, Krasnov BR, Littlewood DTJ (Eds) Parasite diversity and diversification: Evolutionary ecology meets phylogenetics. Cambridge University Press, Cambridge, xiv +488 pp. [ISBN 9781139794749]. https://doi.org/10.1017/CBO9781139794749

- Gilarriortua M, Salona Bordas MI, Cainé L, Pinheiro F, De Pancorbo MM (2013) Cytochrome $b$ as a useful tool for the identification of blowflies of forensic interest (diptera, calliphoridae). Forensic Science International 228 (1-3): 132-136.

https://doi.org/10.1016/j.forsciint.2013.02.037

- Giribet G (2015) Morphology should not be forgotten in the era of genomics-a phylogenetic perspective. Zoologischer Anzeiger-A Journal of Comparative Zoology 256: 96-103. https://doi.org/10.1016/j.jcz.2015.01.003

- Hastriter MW, Bush SE (2006) Maabella gen. nov. (Streblidae: Ascodipterinae) from Guangxi Province, China and Vietnam with notes on preservation of Ascodipterinae. Zootaxa 1176: 27-40. https://doi.org/10.11646/zootaxa.1176.1.3

- Hastriter MW (2007) A review of Ascodipterinae (Diptera: Streblidae) of the Oriental and Australasian regions with a description of three new species of Ascodipteron Adensamer and a key to the subfamily. Zootaxa 1636: 1-32. https://doi.org/10.11646/ zootaxa.1636.1.1

- Jobling B (1939) On the African Streblidae (Diptera Acalypterae) including the morphology of the genus Ascodipteron Adens. and a description of a new species. Parasitology 31: 147-165. https://doi.org/10.1017/S0031182000012701

- Jobling B (1940) Description of the young female and of the male of Ascodipteron africanum Jobling (Diptera, Streblidae). Parasitology 32: 399-404. https://doi.org/ $\underline{10.1017 / S 0031182000015900}$ 
- Jobling B (1952) Description of two new species of Ascodipteron from Africa and one species of Nycteribosca from Madagascar (Diptera, Streblidae). Parasitology 42:

126-135. https://doi.org/10.1017/S0031182000084365

- Jobling B (1956) Streblidae from the French Ivory Coast, with a description of new species (Diptera). Revue Suisse de Zoologie 63: 377-384. https://doi.org/10.5962/ bhl.part.75466

- Jobling B (1958) Streblidae from Yemen with description of one subspecies of Ascodipteron (Diptera). Fieldiana Zoology 39: 185-189.

URL: https://www.biodiversitylibrary.org/page/2848219\#page/7/mode/1up

- Kimura M (1980) A simple method for estimating evolutionary rate of base substitutions through comparative studies of nucleotide sequences. Journal of Molecular Evolution 16: 111-120. https://doi.org/10.1007/BF01731581

- Kumar S, Stecher G, Li M, Knyaz C, Tamura K (2018) MEGA X: Molecular Evolutionary Genetics Analysis across computing platforms. Molecular Biology and Evolution 35: 1547-1549. https://doi.org/10.1093/molbev/msy096

- $\quad$ Kutty SN, Meusemann K, Bayless KM, Marinho MA, Pont AC, Zhou X, et al. (2019) Phylogenomic analysis of Calyptratae: resolving the phylogenetic relationships within a major radiation of Diptera. Cladistics 35 (6): 605-622. https://doi.org/10.1111/cla.12375

- Lavoipierre M (1946) Ascodipteron africanum Job. (Streb. Dipt.) a remarkable South African parasite of bats. Entomological Society of Southern Africa 9: 94-95.

- Liu L, Guo Z, Zhong C, Shi S (2018) DNA barcoding reveals insect diversity in the mangrove ecosystems of Hainan Island, China. Genome 61 (1): 797-806.

https://doi.org/10.1139/gen-2018-0062

- Maa TC (1965) Ascodipterinae of Africa (Diptera: Streblidae). Journal of Medical Entomology 1: 311-326. https://doi.org/10.1093/jmedent/1.4.311

- Misof B, Liu S, Meusemann K, Peters RS, Donath A, Mayer C, et al. (2014) Phylogenomics resolves the timing and pattern of insect evolution. Science 346 (6210): 763-767. https://doi.org/10.1126/science.1257570

- Monticelli F (1898) Di un'altra specie del genere "Ascodipteron" parasita del Rhinolophus clivosus Rüpp. Ricerche fatte nel Laboratorio di Anatomia normale della R. Università di Roma ed in Altri Laboratori Biologici 6 (4): 201-230.

- Muir F (1912) Two new species of Ascodipteron. Bulletin of the Museum of Comparative Zoology 54: 351-366. URL: https://www.biodiversitylibrary.org/page/30209182\#page/ 389/mode/1up

- $\quad$ Pape T, Blagoderov V, Mostovski MB (2011) Order DIPTERA Linnaeus, 1758. Zootaxa 3148: 222-229. https://doi.org/10.11646/zootaxa.3148.1.42

- Petersen FT, Meier R, Kutty SN, Wiegmann BM (2007) The phylogeny and evolution of host choice in the Hippoboscoidea (Diptera) as reconstructed using four molecular markers. Molecular Phylogenetics and Evolution 45 (1): 111-122. https://doi.org/ 10.1016/j.ympev.2007.04.023

- Speiser P (1908) Die Diptera Pupipara der madagassisch-maskarenischen Region. In: Voeltzkow A (Ed.) Reise in Ostafrika in den Jahren 1903-1905: mit Mitteln der Hermann und Elise geb. Heckmann Wentzel-Stiftung: Wissenschaftliche Ergebnisse. E. Scheweizerbart'sche Verlagsbuchhandlung, Stuttgart, vi $+644 \mathrm{pp}$.

- Yan L, Pape T, Elgar MA, Gao Y, Zhang D (2019) Evolutionary history of stomach bot flies in the light of mitogenomics. Systematic Entomology 44: 797-809. https://doi.org/ $\underline{10.1111 / \text { syen. } 12356}$ 
- $\quad$ Yan L, Buenaventura E, Pape T, Kutty SN, Bayless KM, Zhang D (2020) A phylotranscriptomic framework for flesh fly evolution (Diptera, Calyptratae, Sarcophagidae). Cladistics https://doi.org/10.1111/cla.12449

- Zhang D, Yan L, Zhang M, Chu H, Cao J, Li K, et al. (2016) Phylogenetic inference of calyptrates, with the first mitogenomes for Gasterophilinae (Diptera: Oestridae) and Paramacronychiinae (Diptera: Sarcophagidae). International Journal of Biological Sciences 12: 489-504. https://doi.org/10.7150/ijbs.12148 